\title{
STATIC AND DYNAMIC PROPERTIES OF ACTIVE JOINTS
}

\author{
M. Elwenspoek, M. Weustink and R. Legtenberg \\ MESA Research Institute, University of Twente, P.O. Box 217, 7500 AE Enschede, \\ The Netherlands \\ Phone: x31-53-893845 (direct), x31-53-892751 (secretary), Fax x31-53-309547, \\ email: m.elwenspoek@eltn.utwente.nl
}

\begin{abstract}
In this paper the realisation and the first experimental results of a new electrostatic actuator, the 'active joint' are presented. This structure is capable of delivering large forces and large deflections. When a sinusoidal voltage is applied with an amplitude larger than the critical voltage and a frequency close to the resonant frequency, the active joints shows a chaotic response.

A first model is presented in order to describe the dynamic behaviour of active joints.
\end{abstract}

\section{INTRODUCTION}

Electrostatic micro actuators are of considerable interest for microsystems. They are relatively easy to fabricate since in general no special materials (such as piezoelectric or magnetic) are required. However a drawback of electrostatic actuators is the small force or small deflection that can be generated. Combdrives enable us to generate deflections in the order of tens of $\mu \mathrm{m}$ and small forces. By using a larger attractive force between the capacitor plates, the deflection is restricted to a few $\mu \mathrm{m}$. Furthermore, there seems to be no possibility to create larger forces by dielectric materials in these constructions. Here an actuator is presented that, due to its geometry, combines a large force with large displacements (in the order of the linear dimensions of the actuator itself) and which allows to use a dielectric thin films to increase the generated force.

The first description of active joints can be found in [1]. A version moving in the plane of the wafer has been presented by Legtenberg et al. [2]. Here we describe a version for out-of-plane movement. The structure consists of two capacitor plates where one or both are bend. A voltage difference between these plates results in a force which bends the plates to each other (figure 1).

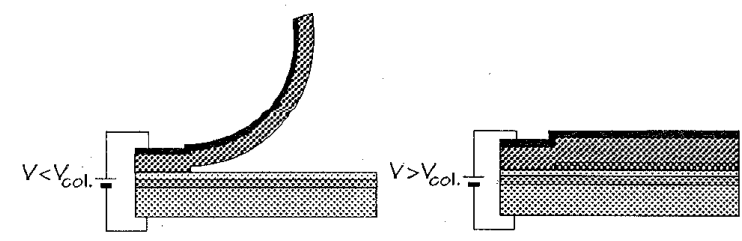

Figure 1. Schematic of an active joint. Left inactivated, right activated.

\section{THEORETICAL MODEL}

\section{Static behaviour}

A first approximation can be made if it is assumed that the actuator is partially collapsed over a length $l$. Then the pull-in voltage is given by:

$$
V_{\text {collapse }}^{2}=\frac{E h^{3}}{12 R^{2}} \frac{d}{\varepsilon} \frac{l b}{A_{e l}}
$$

where $E$ is the effective young's modulus of the cantilever beam material, $h$ its thickness, and $b$ its width. $R$ is the radius of the curvature in initial state. $d$ is the thickness and $\varepsilon$ is the dielectric constant of the insulator. $A_{e l}$ is the area of the ground electrode.

Due to the construction of the actuator the thickness $d$ is not only determined by the thickness of the insulator, but also by the thickness of an air gap. $d$ and $\varepsilon$ can be written as:

$$
\frac{d}{\varepsilon}=\frac{d_{g a p}}{\varepsilon_{g a p}}+\frac{d_{d i e}}{\varepsilon_{d i e}}
$$

Now equation (1) can be written as:

$$
V_{\text {collapse }}^{2}=\frac{E h^{3}}{12 R^{2}}\left(\frac{d_{g a p}}{\varepsilon_{\text {gap }}}+\frac{d_{d i e}}{\varepsilon_{\text {die }}}\right) \frac{l b}{A_{e l}}
$$

The actuator described by equation (3) has only two stable states, however this is not the case when the area $A_{e l}$ depends on $l$. For a triangular ground electrode the collapsed length $l$ will be [1]:

$$
l=L\left[1-\left(\frac{V_{\text {collapse }}}{V}\right)^{2}\right]
$$

where $L$ is the total length of the cantilever beam.

Note that when the actuator is collapsed, $d_{\text {gap }}=0$ so the $V_{\text {collapse }}$ used in equation (4) is given by equation (1), however equation (4) is valid after $V>V_{\text {collapse }}$ as given in equation (3). This is shown in figure 2. 


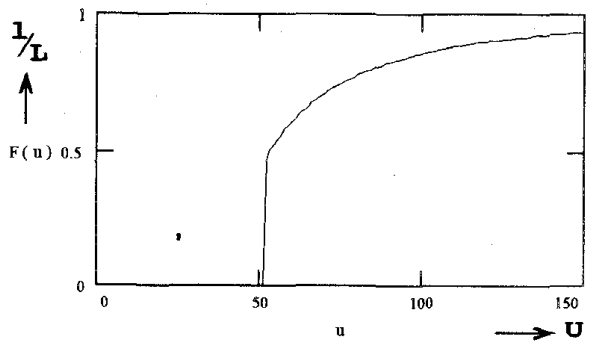

Figure 2. The deflection of the actuator as a function of the applied voltage

\section{Dynamic behaviour}

For the dynamic model the Euler equation has been used:

$$
\frac{d}{d t} \frac{\partial L}{\partial \dot{x}}=-\frac{\partial L}{\partial x}
$$

where $x$ is the length of the collapsed part of the beam and

$$
L=T-U
$$

The potential energy consists of the electrostatic energy $\left(E_{e}\right)$ of the capacitor plates and of the mechanical energy $\left(E_{m}\right)$ of the curved beam, so:

$$
U=E_{e}-E_{m}
$$

where

$$
E_{e}=\varepsilon_{0} \varepsilon_{g a p} \varepsilon_{d i e} b x U^{2}
$$

and

$$
E_{m}=\frac{E h^{3} b|x|}{24 R^{2}}
$$

The absolute value in equation (9) is taken in order to get a equilibrium for $x=0$.

The kinetic energy is given by:

$$
T=\int_{0}^{L-x} A_{b m} \rho V_{p}(p, \dot{x})^{2} d p
$$

where $V_{p}$ equals to the velocity of a infinitesimal strip of the curved beam in point $p$ (figure 3 ), $A_{b m}$ the area and $\rho$ the density of this strip. Due to the movement of the actuator this velocity is directional and the absolute value is given by:

$$
V_{p}(p, v)=2 \sqrt{v^{2} \sin ^{2}\left(\frac{p}{2 R}\right)}
$$

where $v$ equals to the velocity of the centre of the curve i.e. the velocity of the collapsing part of the actuator.

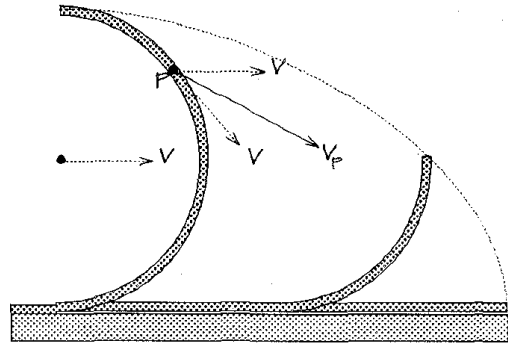

Figure 3. The velocity in a point

With the help of equation (5) trough (11), the following non-linear differential equation can be found:

$$
\begin{aligned}
& |x(t)|^{\prime} \frac{b E h^{3}}{24 R^{2}}-\frac{b \varepsilon_{0} \varepsilon_{g a p} \varepsilon_{d i e}}{2\left(d_{g a p} \varepsilon_{d i e}+\varepsilon_{g a p} d_{d i e}\right)}+ \\
& 3 A_{b m} \rho\left[\cos \left(\frac{L-x(t)}{R}\right)-1\right] \dot{x}^{2}(t)+ \\
& 2 A_{b m} \rho\left[L-R \sin \left(\frac{L-x(t)}{R}\right)-x(t)\right] \ddot{x}(t)=0
\end{aligned}
$$

This equation cannot be solved analytical and has to be evaluated numerically. When this equation is evaluated with the parameters of table 1 , the numerical evaluation gives us the following result (figure 4).

Note that in equation (12) there is no spring constant, i.e. there is no term proportional to $\mathrm{x}$.

\begin{tabular}{|ll|ll|}
\hline$\varepsilon_{0}=8.910^{-6}$ & $R$ & $=16110^{-6}$ \\
$\varepsilon_{\text {die }}=3.8$ & $L$ & $=20010^{-6}$ \\
$\varepsilon_{\text {gap }}$ & $=1$ & $h$ & $=110^{-6}$ \\
$d_{\text {die }}$ & $=5010^{-9}$ & $b$ & $=4010^{-6}$ \\
$d_{\text {gap }}$ & $=21010^{-9}$ & $A_{b m}$ & $=\mathrm{b} \mathrm{h}$ \\
$E$ & $=710^{10}$ & $U$ & $=60$ \\
$\rho$ & $=2.710^{3}$ & $\omega$ & $=1710^{-6}$
\end{tabular}

Table 1. The values used for numerical evaluation

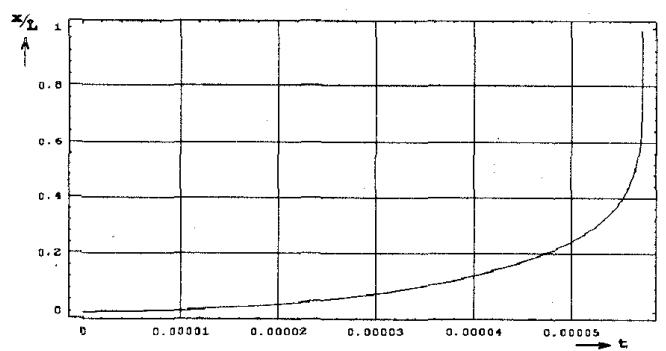

Figure 4. The impact of the actuator after applying a constant voltage 
When instead of a constant voltage, a harmonic voltage is applied then a chaotic behaviour is seen, this is shown in figure 5 .

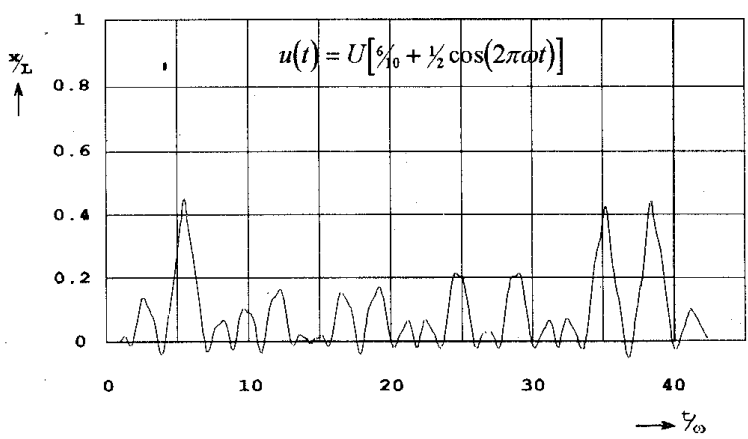

Figure 5a. The displacement of the actuator when a harmonic signal is applied

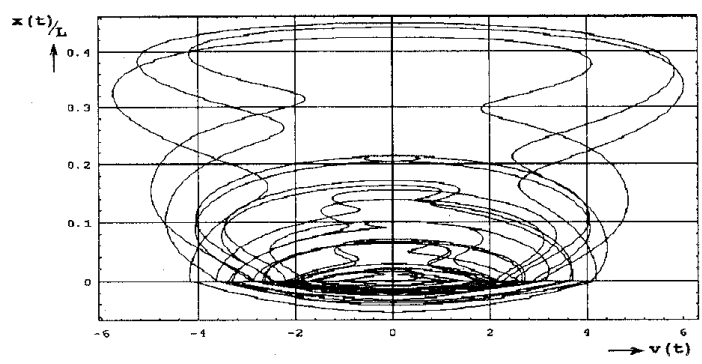

Figure $5 b$. The strange attractor.

\section{FABRICATION}

The active joint described here consists of one curved beam which is fixed at one place and a triangular or rectangular ground electrode separated by an insulator. The realisation of the active joint with the rectangular electrode differs from the method used for the triangular electrode. Both types are realised using micromachining and will be described here.

\section{Rectangular electrode}

After standard wafer cleaning in fuming $\mathrm{HNO}_{3}$ and boiling $\mathrm{HNO}_{3}$, a ground electrode of $\mathrm{Ti} / \mathrm{W}$ is evaporated and the insulating $\mathrm{SiO}_{2}$ layer is deposited using PECVD. Next a sacrificial layer of $20-50 \mathrm{~nm} \mathrm{FC} \mathrm{is} \mathrm{formed} \mathrm{by}$ spincoating at $1500-4000 \mathrm{RPM}$ for $20 \mathrm{~s}$.

After patterning the $\mathrm{FC}$ layer with an $\mathrm{O}_{2}$ plasma a $1 \mu \mathrm{m}$ Al layer and a $50 \mathrm{~nm} \mathrm{Cr}$ layer is evaporated and patterned. Due to the tensile stress in the $\mathrm{Cr} / \mathrm{Al}$ layer and the poor adhesion of the $\mathrm{FC}$, the cantilever beams tend to curl upwards spontaneously. Finally the sacrificial layer is etched using a $\mathrm{O}_{2}$ plasma at $25^{\circ} \mathrm{C}$ for 10 minutes. See figure 6 .

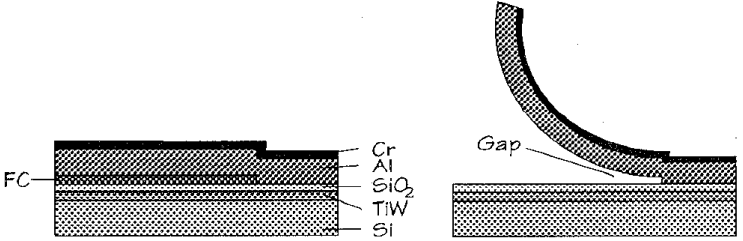

Figure 6. The active joint with rectangular electrode

\section{Triangular electrode}

After standard wafer cleaning, cavities are etched in the wafer. Next the ground electrode of $\mathrm{Ti} / \mathrm{W}$ is evaporated and patterned in the cavities. On a second wafer a thin layer of $1 \mu \mathrm{m}$ glass is sputtered. In order to get sealed cavities both wafers are bonded by using anodic bonding at $450{ }^{\circ} \mathrm{C}$ and backetching in $\mathrm{KOH}$ [3]. After this an $\mathrm{Al}$ layer is evaporated and patterned. Finally the cantilever beams are fried by etching the glass using RIE. See figure 7 and 8.

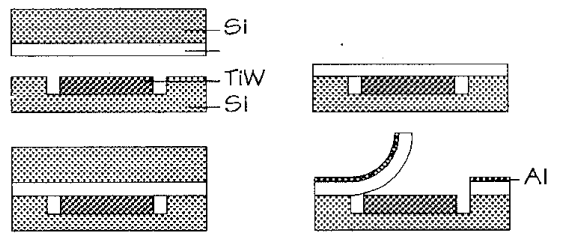

Figure 7. Process steps for a triangular electrode

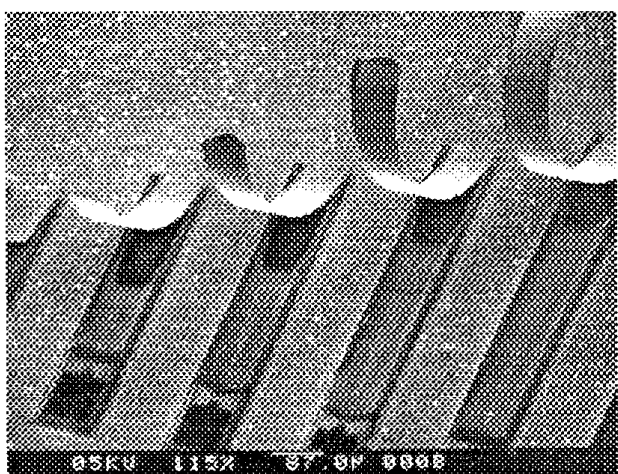

Figure 8. SEM image of an active joint with triangular electrode

\section{EXPERIMENTS}

\section{Static behaviour}

Figure 9 shows optical images of the active joint in different states of excitation. Figure 10 gives a measurement of the deflection of the beam as a function of the voltage. There is a hysteresis if the actuator is fully activated, as is clearly seen in the figure 10 . This might be a result of the lack of an insulator between the ground electrode and the silicon wafer. In this case the silicon wafer acts like an (rectangular) electrode. 
Further it seems as if there were certain stable positions, which probably are due to defects between the beam and the substrate (see figure 8).
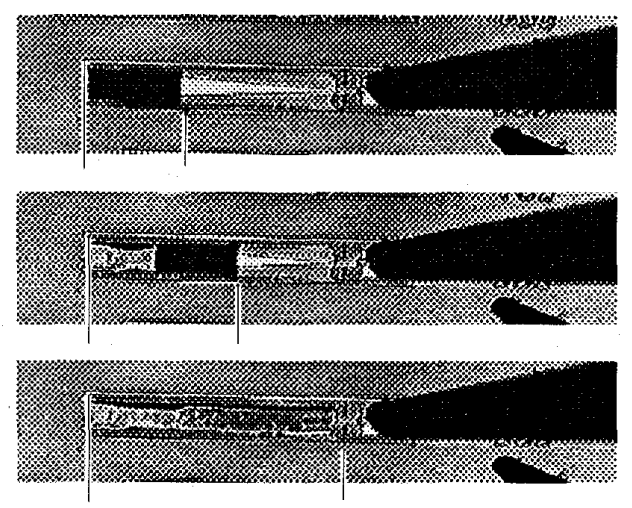

Figure 9. Excitation states of active joints with triangular electrode

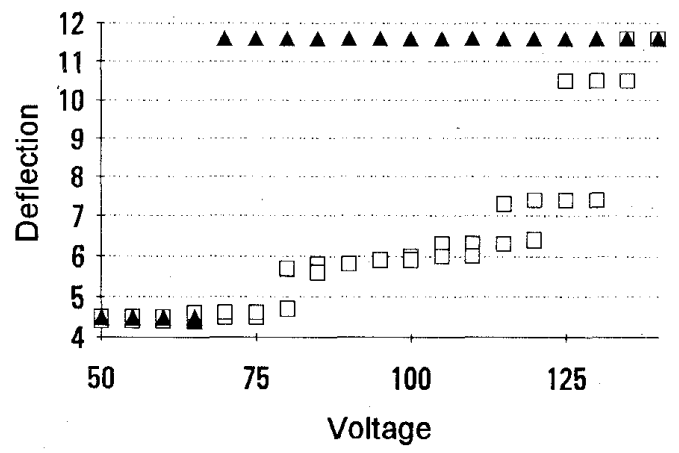

Figure 10. Deflection of the actuator.

A. voltage sweep down, $\square$ : Voltage sweep up.

\section{Dynamic behaviour}

The dynamic properties of the devices have been characterised on optical low magnification microscope with illumination using a stroboscopic lamp. The actuators were excited by sinusoidal waves, triangular waves and block waves, varying the frequency and the amplitude of the voltage. At frequencies below approximately $200 \mathrm{~Hz}$ the beams flipped between their two stable states if the maximum voltage exceeded the critical voltage.

Increasing the frequency up to $250 \mathrm{~Hz}$, the beams started to show erratic behaviour. Instead of switching between the stable states, they sometimes closed, and sometimes opened completely, but in-between they moved with a small amplitude either starting from maximum or minimum positions. Qualitatively, their movement is similar to the plot shown in figure 5a and it seems that there is a certain periodicity. At higher frequencies, the displacements become smaller, but still some 'resonance' frequencies are noticed. We not able to judge if the frequency, at which the chaotic behaviour set in, is related to the fundamental resonance frequency or to a frequency characteristic for the switching time of the actuator, however the model calculations are based on the latter.

The experimental set-up in it's existence now is unable to give more quantitative information over the switching time. Equally, we are not able presently to reproduce phase space images that could reveal the form of the strange attractor like the one in figure $5 \mathrm{~b}$.

\section{DISCUSSION AND CONCLUSIONS}

An electrostatic actuator design has been presented where a cantilever beam can be positioned by means of electrostatic forces. Also a two models are presented to describe the static as well as the dynamic behaviour of the actuator. The dynamics of the actuators are very complex. Close to $V_{\text {collapse }}$ the resonance frequency of the electromechanical system approaches zero as is the case in a parallel plate capacitor close to the pull-in voltage. The dynamic equation is then controlled by a non-linear force, as is the case e.g. in Duffing's equation, see e.g. [4], with consequently bifurcation's and chaos.

Measurements on actuators with a triangular electrode show a voltage controlled deflection, but the behaviour is not in agreement with the theory. This can be explained by the lack of an isolated ground electrode .

Initial experiments on actuators with rectangular electrodes show that the qualitative behaviour is in agreement with the dynamic model. More experiments are required to test the quantitative behaviour, however the calculated 'resonant' frequency is higher then the one observed. When terms of damping and a spring constant are incorporated in the model, better results might be expected. Also the expression for the mechanical energy needs to be reviewed. The energy for $x<0$ should increase much faster than proportional to $x$.

The complex dynamics of the actuator might hamper the application of the device in a microsystem. Presently it seems that the are useful only at small frequencies.

\section{REFERENCES}

[1] M. Elwenspoek, L. Smidt and B. Hök, Active Joints for Microrobot Limbs, J. Micro-mechanics Microeng. 2 (1992) $221-223$

[2] R. Legtenberg, E. Berenschot, M. Elwenspoek and J. Fluitman, Electrostatic curved electrode actuators, Proc. MEMS'95, Amsterdam, The Netherlands, Jan. 29 - Feb. 2 (1995) 37 - 42

[3] J.W. Berenschot, J.G.E. Gardeniers, T.S.J. Lammertink and M. Elwenspoek, New applications of r.f.-sputtered glass films as protection and bonding layers in silicon micromachining, Sensors and Actuators A, 41-42 (1994) 338-343

[4] J.M.T. Thompson and H.B. Steward, Non-linear Dynamics and Chaos, John Wiley, New York (1986). 